\title{
Photoneutron reaction cross sections from various experiments - analysis and evaluation using physical criteria of data reliability
}

\author{
Vladimir Varlamov ${ }^{1,}$, , Boris Ishkhanov ${ }^{1,2}$, Vadim Orlin $^{1}$, Nikolai Peskov $^{1}$, and Mikhail Stepanov ${ }^{2}$ \\ ${ }^{1}$ Lomonosov Moscow State University, Skobeltsyn Institute of Nuclear Physics, 119991 Moscow, Russia \\ 2 Lomonosov Moscow State University, Physics Department, 119991 Moscow, Russia
}

\begin{abstract}
The majority of photonuclear reaction cross sections important for many fields of science and technology and various data files (EXFOR, RIPL, ENDF, etc.) supported by the IAEA were obtained in experiments using quasimonoenergetic annihilation photons. There are well-known systematic discrepancies between the partial photoneutron reactions $(\gamma, 1 \mathrm{n}),(\gamma, 2 \mathrm{n}),(\gamma, 3 \mathrm{n})$. For analysis of the data reliability the objective physical criteria were proposed. It was found out that the experimental data for many nuclei are not reliable because of large systematic uncertainties of the neutron multiplicity sorting method used. The experimentally-theoretical method was proposed for evaluating the reaction cross sections data satisfying the reliability criteria. The partial and total reaction cross sections were evaluated for many nuclei. In many cases evaluated data differ noticeably from both the experimental data and the data evaluated before for the IAEA Photonuclear Data Library. Therefore it became evident that the IAEA Library needs to be revised and updated.
\end{abstract}

\section{Introduction}

Photonuclear data, first of all cross sections of total and partial photoneutron reactions with different numbers of outgoing particles, are widely used in many fields of both basic and applied research.

The majority of data for the partial $(\gamma, 1 \mathrm{n}),(\gamma$, $2 \mathrm{n}),(\gamma, 3 \mathrm{n})$ and the total $(\gamma$, tot $)=(\gamma, 1 \mathrm{n})+(\gamma$, $2 \mathrm{n})+(\gamma, 3 \mathrm{n})$ photoneutron reactions were obtained at Livermore (USA) and Saclay (France) using the quasimonoenergetic annihilation photon beams and the photoneutron multiplicity sorting method [1]. There are well-known significant (up to 100\%) discrepancies between the results of those two laboratories for the same nuclei [2-4]. Those discrepancies are clearly systematic: as a rule $(\gamma, 1 \mathrm{n})$ reaction cross sections have larger values at Saclay but $(\gamma, 2 \mathrm{n})$ reaction cross sections vice versa at Livermore.

The method for putting Livermore and Saclay data into consistency was proposed [2] on the base of comparison ${ }^{181} \mathrm{Ta}(\gamma, 2 \mathrm{n}){ }^{179} \mathrm{Ta}$ reaction cross sections obtained at Livermore and Saclay using the neutron multiplicity sorting method with correspondent data obtained using the alternative activation method. It was concluded that Livermore data were correct but Saclay data were underestimated. In particular, it was recommended to recalculate both $(\gamma, 1 \mathrm{n})$ and $(\gamma, 2 \mathrm{n})$ unreliable Saclay data using appropriate part of the neutron yield reaction cross sections close at both laboratories. For 19 nuclei

a e-mail: vVVarlamov@gmail.com from ${ }^{51} \mathrm{~V}$ to ${ }^{238} \mathrm{U}$ data were jointly evaluated at the Russia Lomonosov Moscow State University Skobeltsyn Institute of Nuclear Physics Centre for Photonuclear Experiments (CDFE) [4]. Previously "unreliable" Saclay data were corrected to schieve consistency with "reliable" Livermore data.

At the same time one can see many physically forbidden negative values in the $(\gamma, 1 \mathrm{n})$ reaction cross sections obtained at Livermore for various nuclei $\left({ }^{94} \mathrm{Zr}\right.$, ${ }^{115} \mathrm{In},{ }^{116} \mathrm{Sn},{ }^{159} \mathrm{~Tb},{ }^{188} \mathrm{Os},{ }^{208} \mathrm{~Pb}$ and others) at the photon energies above the $(\gamma, 2 n)$ reaction threshold $B_{2 n}$. Three typical examples are presented in Fig. 1. Therefore there are serious doubts that Livermore data in many cases are really reliable.

For analysis of the data under discussion reliability the special objective physical criteria and new experimentallytheoretical method for reliable data evaluating were proposed. New partial and total photoneutron reactions cross sections for nuclei ${ }^{63,65} \mathrm{Cu},{ }^{80} \mathrm{Se},{ }^{89} \mathrm{Y},{ }^{91,94} \mathrm{Zr}$, ${ }^{115} \mathrm{In},{ }^{112-124} \mathrm{Sn},{ }^{133} \mathrm{Cs},{ }^{159} \mathrm{~Tb},{ }^{181} \mathrm{Ta},{ }^{186}{ }^{-192} \mathrm{Os},{ }^{197} \mathrm{Au}$, ${ }^{208} \mathrm{~Pb},{ }^{209} \mathrm{Bi}$ were evaluated. Data satisfying the proposed reliability criteria in many cases differ noticeably from both the experimental data and the data evaluated before for the IAEA Photonuclear Data Library using codes GNASH, ALICE-F, GUNF, GLANF [8].

Therefore it became evident that the IAEA Library needs to be revised and updated. With this aim the IAEA Coordinated Research Program (F41032, Research Contract 20501) on Updating the Photonuclear data Library and Generating a Reference Database for Photon Strength Functions was adopted for period 2016-2018. 


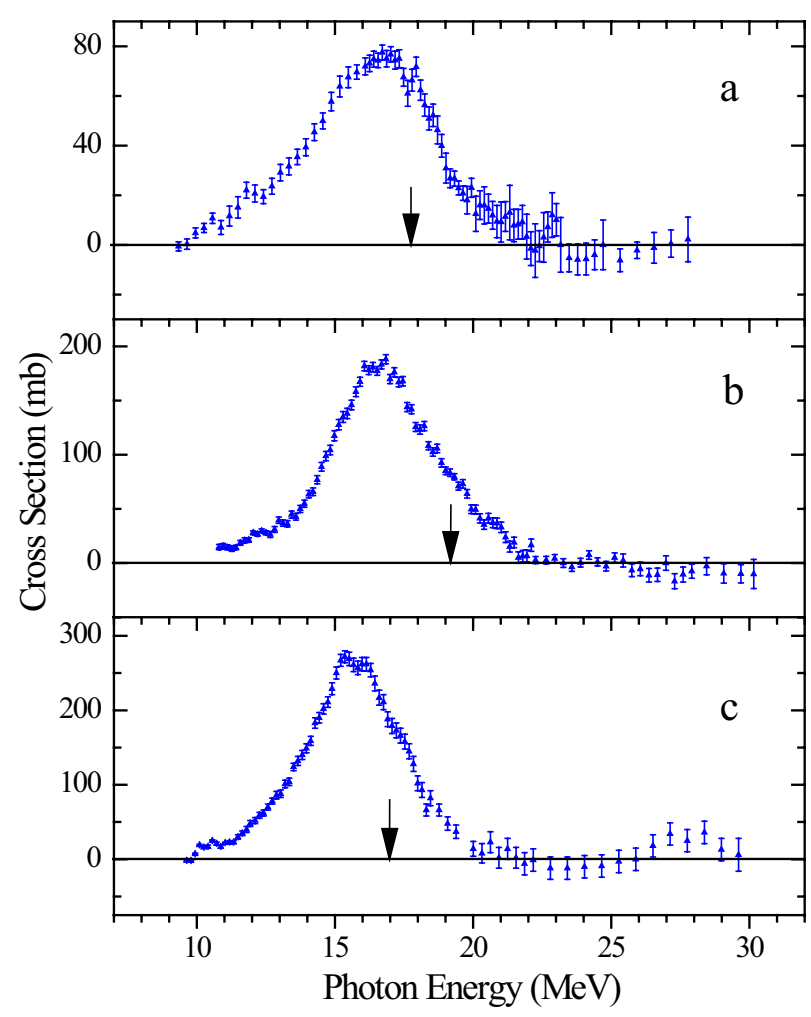

Figure 1. Unreliable physically forbidden negative values in the $(\gamma, 1 \mathrm{n})$ reaction cross sections obtained at Livermore: $\mathrm{a}-{ }^{65} \mathrm{Cu}[5]$; b- ${ }^{91} \mathrm{Zr}$ [6]; c- ${ }^{116} \mathrm{Sn}$ [7]. Arrows mark $\mathrm{B}_{2 n}$ values.

\section{The physical criteria of partial photoneutron reaction cross section reliability}

The simple and clear objective physical criteria of partial photoneutron reaction cross section data reliability were proposed [9]: the ratios $\mathrm{F}_{\mathrm{i}}(\gamma$, in $)=\sigma(\gamma$, in $) / \sigma(\gamma, \mathrm{Sn})$, where $(\gamma$, in) is the partial reaction with neutron multiplicity "i" and $(\gamma, \mathrm{Sn})=(\gamma, 1 \mathrm{n})+2(\gamma, 2 \mathrm{n})+3(\gamma$, $3 n)$ is the neutron yield reaction.

Follow definitions $F_{i}$ could not have values higher than $1.00,0.50,0.33$ correspondingly for $\mathrm{i}=1,2,3$. Larger values mean that experimental neutron multiplicity sorting was carried out with significant systematic uncertainties and therefore cross sections obtained are not reliable.

It was found out that unreliable negative $(\gamma$, 1n) reaction cross section (and negative $F_{1}$ values correspondingly) values clearly correlate with unreliable values $\mathrm{F}_{2}>0.50$.

The typical examples of $F_{1,2}$ energy dependencies for ${ }^{116} \mathrm{Sn}$ data obtained at Livermore [7] and Saclay [10] are presented in Fig. 2 in comparison with the results of correspondent calculations in the combined model of photonuclear reactions [11]. The preequilibrium model is based on the Fermi-gas densities equations and takes into account the effects of nucleus deformation and nucleus giant dipole resonance (GDR) isospin splitting. The model is well-tested for description of photoneutron yield reaction cross sections for medium and heavy nuclei.

One can see that in the energy range $\sim 21-30 \mathrm{MeV}$ data obtained at Livermore are not reliable. The reason
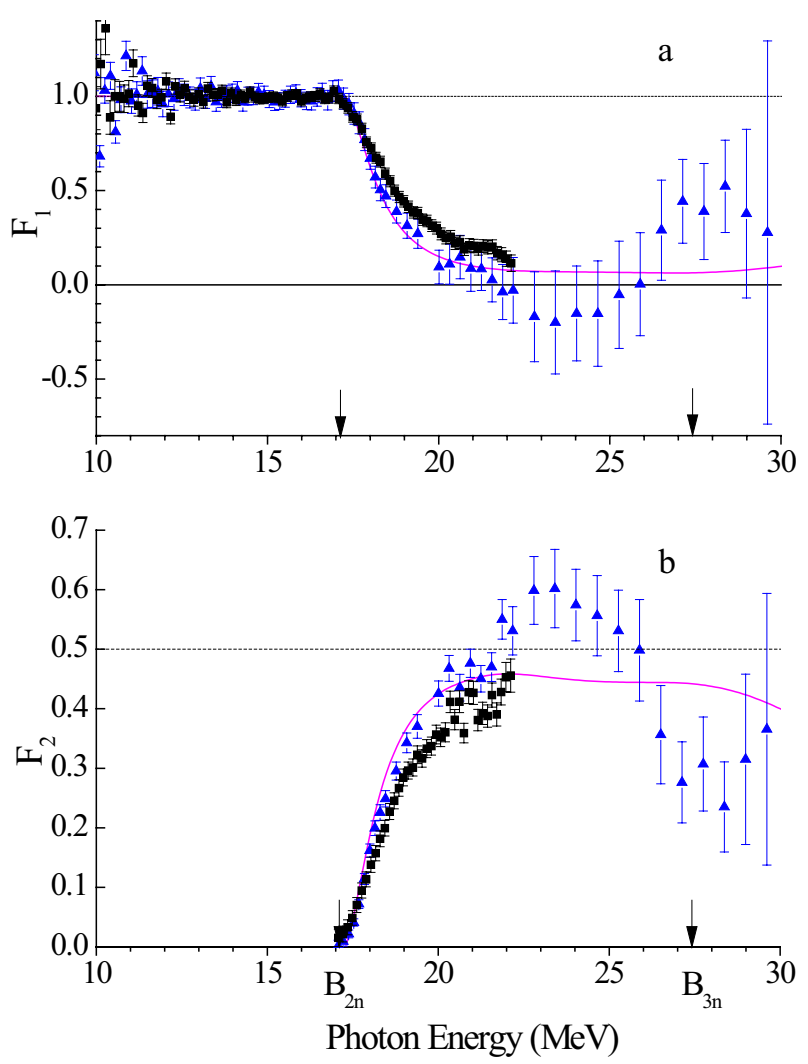

Figure 2. The data reliability criteria ratios $F_{1}$ (a) and $F_{2}$ (b) for ${ }^{116} \mathrm{Sn}$ obtained for experimental cross sections: triangles Livermore data [7], squares - Saclay data [10], lines - results of calculations in model [11].

Table 1. Negative $\sigma(\gamma, 1 \mathrm{n})$ and $\mathrm{F}_{1}$ and $\mathrm{F}_{2}>0.50$ values.

\begin{tabular}{|c|c|c|}
\hline Nucleus [Ref.] & $\begin{array}{c}\text { Negative } \sigma(\gamma, 1 \mathrm{n}) \text { and } \mathrm{F}_{1} \\
\text { values energy range, } \mathrm{MeV}\end{array}$ & $\begin{array}{c}\mathrm{F}_{2} \text { maximal } \\
\text { value }\end{array}$ \\
\hline${ }^{91} \mathrm{Zr}[6]$ & $\sim 23.0-30.0$ & $\sim 0.80$ \\
\hline${ }^{94} \mathrm{Zr}[6]$ & $\sim 21.5-27.0$ & $\sim 0.70$ \\
\hline${ }^{115} \mathrm{In}[6]$ & $\sim 20.5-31.0$ & $\sim 0.60$ \\
\hline${ }^{116} \mathrm{Sn}[7]$ & $\sim 21.5-26.0$ & $\sim 0.62$ \\
\hline${ }^{159} \mathrm{~Tb}[12]$ & $\sim 18.5-22.0$ & $\sim 0.60$ \\
& $\sim 22.5-24.0$ & $\sim 2.00$ \\
\hline
\end{tabular}

could be the incorrect assignment of neutrons to the $(\gamma, 2 \mathrm{n})$ channel instead of the $(\gamma, 1 \mathrm{n})$ channel and to the $(\gamma, 1 \mathrm{n})$ channel instead of $(\gamma, 3 n)$ channel, respectively, because of errors in neutron multiplicity determination using its kinetic energy measuring [1] based on the supposition that energy of neutron from $(\gamma, 1 \mathrm{n})$ reaction is larger than energies of both neutrons from $(\gamma, 2 \mathrm{n})$ reaction.

The similar experimental energy dependencies for the ratios $F_{1,2}$ were obtained using correspondent experimental data for many nuclei mentioned above. Some examples of unreliable data for $(\gamma, 1 \mathrm{n})$ and $(\gamma, 2 \mathrm{n})$ reaction cross sections obtained at Livermore are presented in Table 1.

The ratios $\mathrm{F}_{3}$ for many nuclei (if $(\gamma, 3 \mathrm{n})$ reaction cross sections were obtained) in various energy ranges also result in unreliable $\left(\mathrm{F}_{3}<0.00\right.$ or $\left.\mathrm{F}_{3}>0.33\right)$ values. It means that the sorting of neutrons between " $1 n-3 n$ " and " $2 n-3 n$ " channels also was not reliable. 


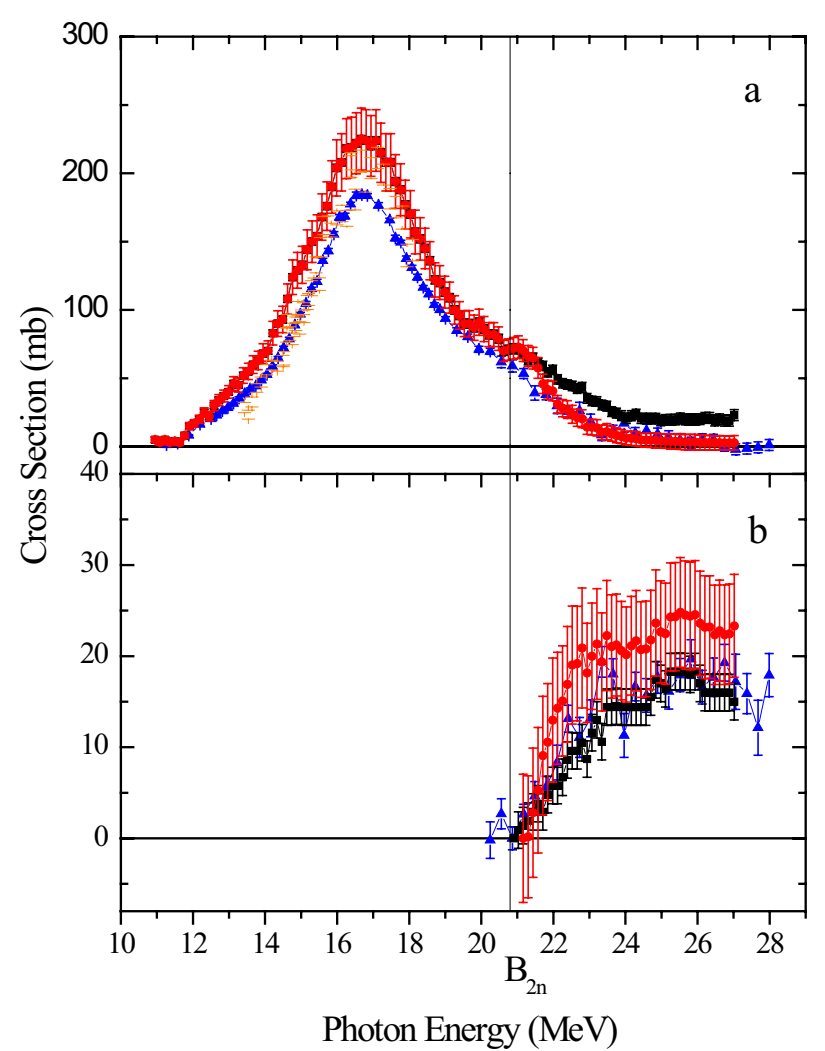

Figure 3. Comparison of the evaluated (circles) and the experimental (triangles [6], squares [13]) partial reaction cross sections for ${ }^{89} \mathrm{Y}$ : $\mathrm{a}-(\gamma, 1 \mathrm{n}), \mathrm{b}-(\gamma, 2 \mathrm{n})$.

\section{The experimentally-theoretical method for reliable partial photoneutron reaction cross section evaluating}

Using the data reliability criteria described above the new method for evaluating the reliable partial photoneutron reaction cross sections were proposed [9]: $\sigma^{\text {eval }}(\gamma$, in $)=$ $\mathrm{F}_{\mathrm{i}}^{\text {theor }}(\gamma$, in $) \times \sigma^{\exp }(\gamma, \mathrm{Sn})$.

This treatment is based on the results of the analysis [4] of large systematics of the disagreements between experimental data for $(\gamma, \mathrm{Sn})$ reaction not dependent on the neutron multiplicity. It was shown that disagreements between $\sigma^{\exp }(\gamma, \mathrm{Sn})$ obtained in various experiments is relatively small $(\sim 10 \%)$.

Therefore the new experimentally-theoretical method for partial photoneutron reaction cross section evaluating means that specially selected evaluated preliminarily experimental photoneutron yield reaction cross section $\sigma^{\exp }(\gamma, \mathrm{Sn})$ sorting is separated into partial reaction parts $\sigma^{\text {eval }}(\gamma$, in $)$ in accordance with the equations of the combined model of photonuclear reactions [11]. The partial reactions cross sections for $(\gamma, 1 \mathrm{n}),(\gamma, 2 \mathrm{n})$, and $(\gamma$, $3 n)$ calculated in the model were used for determination of the ratios $\mathrm{F}_{\mathrm{i}}^{\text {theor }}(\gamma$, in).

\section{The disagreements between new evaluated and experimental data}

The evaluated partial photoneutron reaction cross sections obtained for many nuclei mentioned above in many cases noticeably differ from the correspondent experimental cross sections. The typical example $\left({ }^{89} \mathrm{Y}\right)$ is presented in Fig. 3.

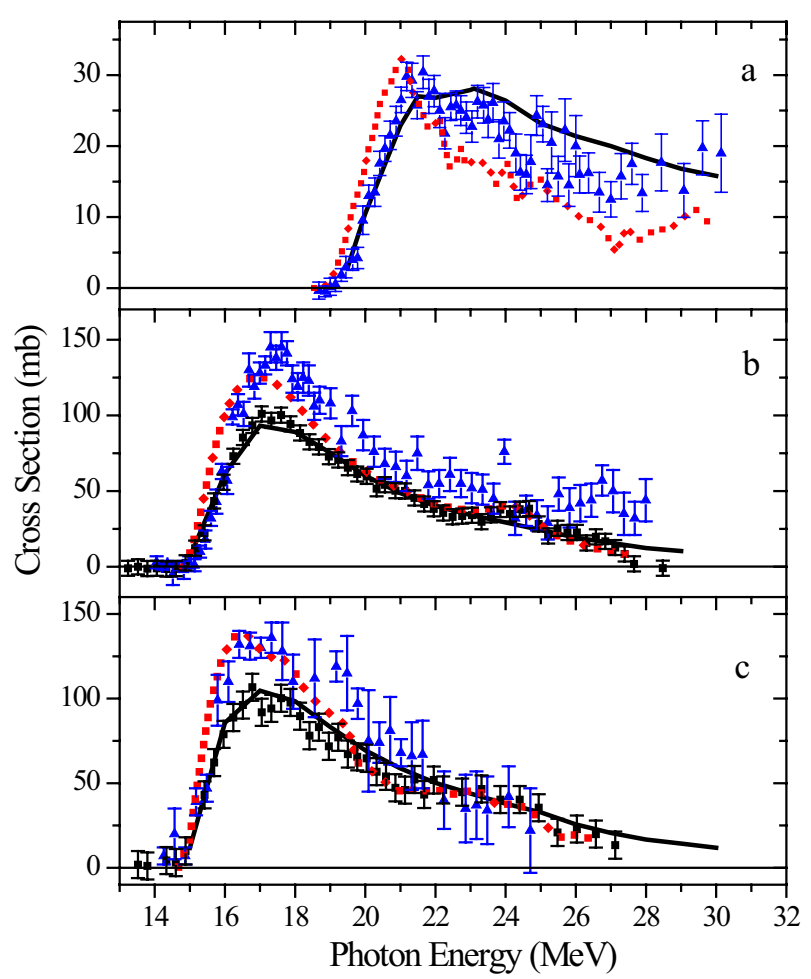

Figure 4. Comparison of the old (solid lines) and the new (dots) evaluated $(\gamma, 2 \mathrm{n})$ reaction cross sections with experimental data: a- ${ }^{91} \mathrm{Zr}$, (triangles [6], solid line [8], dots [16]); b- ${ }^{159} \mathrm{~Tb}$ (triangles [12], squares [15], solid line [8], dots [14]); c- ${ }^{197} \mathrm{Au}$ (triangles [17], squares [18], solid line [8], dots [9]).

One can see that in the energy range below $\sim \mathrm{B}_{2 \mathrm{n}}$ the evaluated $(\gamma, 1 \mathrm{n})$ reaction cross section is in agreement with Saclay data but at larger energies vice versa with Livermore data. The evaluated $(\gamma, 2 \mathrm{n})$ reaction cross section has a larger values in comparison with both experimental cross sections. One can see that disagreements under discussion have values about tens of $\mathrm{mb}$.

In [14] it was shown for ${ }^{159} \mathrm{~Tb}$ that in the energy range between thresholds $\mathrm{B}_{2 n}$ and $\mathrm{B}_{3 n}$ integrated cross section $\sigma^{\text {eval-int }}(\gamma, 1 \mathrm{n})$ is about $20 \%$ larger than the Livermore data [12] and about $20 \%$ smaller than the Saclay measurements [15], whereas $\sigma^{\text {eval-int }}(\gamma, 2 \mathrm{n})$ is $15 \%$ larger than in [15] and $20 \%$ smaller as compared to those of [12]. Therefore the difference between evaluated and experimental $[12,15]$ values of the important cross section ratio $\sigma^{\text {eval-int }}(\gamma, 2 \mathrm{n}) / \sigma^{\text {eval-int }}(\gamma, 1 \mathrm{n})$ is $\sim 30 \%$.

Additional examples of the noticeable disagreements between evaluated and experimental data for ${ }^{91} \mathrm{Zr},{ }^{159} \mathrm{~Tb}$ and ${ }^{197} \mathrm{Au}$ are presented in Fig. 4.

\section{The disagreements between the new and old evaluated data}

One can see from Fig. 4 that the partial photoneutron reaction cross sections evaluated using the method described above noticeably differ not only from the experimental data but also from the correspondent evaluated cross sections from the IAEA Photonuclear Data Library obtained before [15]. Three examples for ${ }^{91} \mathrm{Zr}$, ${ }^{159} \mathrm{~Tb}$ and ${ }^{197} \mathrm{Au}$ are presented in Fig. 4. 
As was mentioned in the Introduction old evaluations were carried out using codes GNASH, ALICE-F, GUNF, GLANF [8], as a rule in order to model accurately Saclay cross section data for reaction $(\gamma$, tot $)=(\gamma, 1 \mathrm{n})+$ $(\gamma, 2 n)+(\gamma, 3 n)$. Therefore the possible reason for disagreements under discussion could be that previous evaluations [15] were based on the total photoneutron reaction $(\gamma$, tot) instead of neutron yield reaction $(\gamma, \mathrm{Sn})$ cross sections. If partial reaction cross sections contain noticeable systematic uncertainties their sum also can contain systematic uncertainties.

\section{The IAEA coordinated research program}

Because of the reasons described it became evident that IAEA Library needs to be revised and updated.

The correspondent Coordinated Research Program "Updating the Photonuclear data Library and Generating a Reference Database for Photon Strength Functions“ “ [19] was proposed and adopted by the International Atomic Energy Agency.

It addition to the data obtained before $[9,11,14,16,20$, 21] many new photoneutron reaction cross sections will be evaluated using the experimentally-theoretical method and data reliability criteria described.

The new partial photoneutron reaction cross sections will be evaluated for nuclei experimental data for which are noticeably doubtful and for those for which evaluations have not be done during the previous IAEA CRP [8]. In addition to nuclei mentioned above new data will be obtained for ${ }^{103} \mathrm{Rh},{ }^{133} \mathrm{Cs},{ }^{138} \mathrm{Ba},{ }^{140,142} \mathrm{Ce},{ }^{141} \mathrm{Pr},{ }^{145,148} \mathrm{Nd}$, ${ }^{153} \mathrm{Eu},{ }^{160} \mathrm{Gd},{ }^{186} \mathrm{~W}$, and some others.

The new evaluated partial photoneutron reaction cross sections will be compared with the new experimental data obtained using various modern facilities, first of all laser Compton-backscattering $\gamma$-rays facility NewSubaru (Japan). The yields of multi-nucleon reactions measured by activation method on the Skobeltsyn Institute of Nuclear Physics racetrack microtron also will be used. The new data will be used for updating the IAEA Photonuclear Data Library.

The work was supported by Russia FBR Grant 13-02-00124 and is supported by the IAEA CRP (F41032, RC 20501) on Updating the Photonuclear data Library and Generating a Reference Database for Photon Strength Functions.

\section{References}

[1] S.S. Dietrich, B.B. Berman, At. Data Nucl. Data Tables. J. 38, 199 (1988)
[2] E. Wolynec, M.M. Martins, Rev. Bras. Phys. J. 17, 56 (1987)

[3] B.L. Berman, R.E. Pywell, S.S. Dietrich, M.N. Thomson, K.G. McNeil, J.W. Jury, Phys. Rev. J. C 36, 1286 (1987)

[4] V.V. Varlamov, N.N. Peskov, D.V. Rudenko, M.E. Stepanov, INDC(CCP)-440, IAEA NDS, Vienna, Austria, p. 37 (2004)

[5] S.C. Fultz, R.L. Bramblett, J.T. Caldwell, R.R. Harvey, Phys. Rev. J. 133, B1149 (1964)

[6] B.L. Berman, J.T. Caldwell, R.R. Harvey, M.A. Kelly, R.L. Bramblett, S.C. Fultz, Phys. Rev. J. 162, 1098 (1967)

[7] S.C. Fultz, B.L. Berman, J.T. Caldwell, R.L. Bramblett, M.A. Kelly, Phys. Rev. J. 186, 1255 (1969)

[8] Handbook on photonuclear data for applications. Cross-sections and spectra. IAEA-TECDOC-1178, 2000

[9] V.V. Varlamov, B.S. Ishkhanov, V.N. Orlin, S.Yu. Troshchiev, Bull. Rus. Acad. Sci. Phys. J. 7, 842 (2010)

[10] A. Lepretre, H. Beil, R. Bergere, P. Carlos, A. De Miniac, A. Veyssiere, K. Kernbach, Nucl. Phys. J. A 219, 39 (1974)

[11] B.S. Ishkhanov, V.N. Orlin, Phys. Atom. Nucl. J. 71, 493 (2008)

[12] R.L. Bramblett, J.T. Caldwell, R.R. Harvey, S.C. Fultz, Phys. Rev. J. 133, B869 (1964)

[13] A. Lepretre, H. Beil, R. Bergere, P. Carlos, A. Veyssiere, M. Sugawara, Nucl. Phys. J. A 175, 609 (1971)

[14] V.V. Varlamov, B.S. Ishkhanov, V.N. Orlin, K.A. Stopani, Eur. Phys. J. Hadr. and Nucl. J. A 50, 114 (2014)

[15] R. Bergere, H. Beil, A. Veyssiere, Nucl. Phys. J. A 121, 463 (1968)

[16] V.V. Varlamov, M.A. Makarov, N.N. Peskov, M.E. Stepanov, Phys. Atom. Nucl. J. 78, 634 (2015)

[17] S.C. Fultz, R.L. Bramblett, T.J. Caldwell, N.A. Kerr, Phys. Rev. J. 127, 1273 (1962)

[18] A. Veyssiere, H. Beil, R. Bergere, P. Carlos, A. Lepretre, Nucl.Phys. J. A 159, 561 (1970)

[19] P. Dimitriou, R.B. Firestone, S. Siem, F. Becvar, M. Krticka, V.V. Varlamov, M. Wieddeking, EPJ Web of Conferences 93, 06004 (2015)

[20] V.V. Varlamov, B.S. Ishkhanov, V.N. Orlin, N.N. Peskov, Phys. Atom. Nucl. J. 79, 501 (2016)

[21] V.V. Varlamov,A.I. Davydov, M.A. Makarov, V.N. Orlin, N.N. Peskov, Bull. Rus. Acad. Sci. Phys. J. 80, 317 (2016) 